\title{
Species richness of the genera Trachelomonas and Strombomonas (pigmented Euglenophyceae) in a subtropical urban lake in the Porto Alegre Botanical Garden, RS, Brazil
}

Sandra Maria Alves-da-Silva ${ }^{1,3}$, Josemari da Cruz Cabreira ${ }^{1}$, José Guilherme Voos² and Eduardo Alcayaga Lobo ${ }^{2}$

Received: 31 January, 2012. Accepted: 9 May, 2013

\begin{abstract}
The objective of this study was to evaluate the taxonomic composition and richness of the genera Trachelomonas and Strombomonas (pigmented Euglenophyceae) in a subtropical urban lake $\left(30^{\circ} 03^{\prime} 05^{\prime \prime} \mathrm{S} ; 51^{\circ} 10^{\prime} 34^{\prime \prime} \mathrm{W}\right)$ within the Botanical Garden, in the city of Porto Alegre, located in the state of Rio Grande do Sul, Brazil, in order to contribute to the floristic knowledge of Euglenophyta in artificial urban lakes. From July 2007 to June 2008, we collected samples monthly from two sampling stations, using a $25-\mu \mathrm{m}$ mesh plankton net. The samples were preserved in $4 \%$ formaldehyde. We evaluated the following environmental variables related to the water in the lake: ammonia content, silica content, organic matter content, dissolved oxygen concentration, temperature, $\mathrm{pH}$, depth, transparency, electrical conductivity and rainfall. To study the species composition in relation to environmental factors, we used cluster analysis, principal components analysis, canonical correspondence analysis and indicator species analysis. At the specific and infraspecific level, 22 taxa were identified, Trachelomonas elliptica (Playf.) Defl. and T. gracillima Bal. \& Dast. representing new records for the state of Rio Grande do Sul. The cluster analysis indicated seasonal variation, species richness being highest in the spring of 2007. Seven indicator species were characterized as acid-tolerant.
\end{abstract}

Key words: Euglenophyta, environmental gradient, shallow lake, species richness, southern Brazil

\section{Introduction}

Euglenophyceae is a class of microalgae composed of pigmented and colorless organisms, which, except for the genus Colacium, are all single-celled flagellates found in moist soil, brackish water, fresh water and marine environments. The pigmented Euglenophyta of the order Euglenales have two flagella, and only one emerges from the reservoir at the anterior pole of the cell, they show a pellicle composed of protein striae and have an energy reserve of paramylon. The pigmented genera Trachelomonas Ehr emend. Defl. and Strombomonas Defl. were studied in the Lago da Ponte, which is an artificial urban lake within the Botanical Garden, in the city of Porto Alegre, located in the state of Rio Grande do Sul, Brazil. Trachelomonas is the genus with the largest number of species within the division Euglenophyta, encompassing approximately 250 species that inhabit freshwater environments worldwide (Bourrelly, 1970), including marine species (Leedale, 1967), whereas Strombomonas encompasses approximately 50 species found only in freshwater environments (Bourrelly, 1970).

The Porto Alegre Botanical Garden, with an area of 39 ha, is located within an urban area and includes two lakes known locally as the Lago das Tartarugas ("Turtle Lake") and Lago da Ponte ("Bridge Lake"). We studied the Lago da Ponte, which was previously a swamp. Through the removal of grasses and the addition of more water, it was transformed into a lake in 2003. It is a shallow lake with grass carp, two swans and a number of tortoises. In its reflecting pool, Salvinia auriculata Abl. can be found. The decomposition of aquatic macrophytes associated with the food fed to swans and animal feces propitiate the existence of Euglenophyta, a group of algae that has been widely cited as a major indicator of water with high concentrations of organic matter, nitrogen and phosphorus (Round, 1983).

Urban lakes differ greatly in size, depth, drainage, water volume, hydrological balance, nutrient cycling and trophic status (García-Gil \& Camacho, 2001). Although artificial urban lakes present environmental conditions suitable for supporting a highly diverse group of Euglenophyta, there have been few studies of these biotopes in the state of Rio Grande do Sul, although there have been some notable studies conducted by Rosa (1974), Cecy (1976; 1986; 1990), Huzsar et al. (1990), Franceschini (1992), Alves-da-Silva \& Torres (1992; 1994a; 1994b; 1994c), Alves-da-Silva \& Avila (1995) and Domingues \& Torgan (2011). In Brazil as a whole,

\footnotetext{
${ }^{1}$ Fundação Zoobotânica do Rio Grande do Sul, Museu de Ciências Naturais, Porto Alegre, RS, Brazil

${ }^{2}$ Universidade de Santa Cruz do Sul, Laboratório de Limnologia, Santa Cruz do Sul, RS, Brazil

${ }^{3}$ Author for correspondence: alvesdasilva@fzb.rs.gov.br
} 
studies of Euglenophyta in urban lakes or ponds include those conducted by Heckmann et al. (1993), Jati \& Train (1994), Branco \& Senna (1996), Giani et al. (1999), Nogueira \& Rodrigues (1999), Silva (1999), Ferreira \& Menezes (2000), Goulart et al. (2002), Kepeller et al. (2002), Ferragut et al. (2005), Nogueira et al. (2008) and Tucci et al. (2006). In this context, in order to bridge the knowledge gap on biodiversity of this group of algae in artificial urban ponds in the state and country, the objective of the present study was to study the taxonomic composition and richness of the genera Trachelomonas and Strombomonas, as well as their relationship to environmental variables, in the Lago da Ponte.

\section{Material and methods}

\section{Study area and sampling}

The geographic coordinates of the Lago da Ponte are $30^{\circ} 03^{\prime} 05^{\prime \prime} \mathrm{S}$ and $51^{\circ} 10^{\prime} 34^{\prime \prime} \mathrm{W}$. It is an artificial shallow water environment with an approximate area of $0.5 \mathrm{ha}$. We collected samples of Trachelomonas and Strombomonas species at two locations: Station 1, with an average depth of $54.1 \mathrm{~cm}$ (max., $100 \mathrm{~cm}$ ); and Station 2, with an average depth of $22.8 \mathrm{~cm}$ (max., $30 \mathrm{~cm}$ ). From July 2007 to June 2008 encompassing all four seasons, monthly samples were collected from the two sampling stations, totaling 24 samplings. Operationally, the nomenclature used hereafter is: $\mathrm{E} 1$ and $\mathrm{E} 2=$ sampling Stations 1 and 2; 77 = month $/$ year (July 2007); E177 = Station 1, July 2007; E277 = Station 2, July 2007; E197 = Station 1, September 2007; E297 = Station 2, September 2007, and so on.

\section{Biological composition}

The water samples for biological analyses were collected using a $25-\mu \mathrm{m}$ mesh plankton net and were preserved in $4 \%$ formaldehyde. We analyzed slide-mounted live samples using a light microscope (Leica DMLS; Leica Microsystems. Inc., Depew, NY, USA) with a grid eyepiece, which was coupled to a camera lucida in order to make the drawings.

For the identification of taxa at the specific and infraspecific levels, we referred to the following basic works: Huber-Pestalozzi (1955), Németh (1980), Starmach (1983), Tell \& Conforti (1986) and Shi et al. (1999). The samples were deposited in the collection of the Herbário Prof. Dr. Alarich R. H. Schultz (HAS, Prof. R. Alarich H. Schultz Herbarium) at the Museum of Natural Science of the Zoobotanical Foundation of the state of Rio Grande do Sul (Tab. 1).

\section{Environmental variables}

Simultaneously to the collection of biological material, water was collected in order to conduct measurements of physical and chemical variables important as parameters for environmental evaluation. The concentrations of ammonia $\left.(\mu \mathrm{g} \mathrm{L})^{-1}\right)$, silica $\left(\mathrm{mg} \mathrm{L}^{-1}\right)$, organic matter $\left(\mathrm{mg} \mathrm{L}^{-1} \mathrm{O}_{2}\right)$ and dis- solved oxygen $\left(\mathrm{mg} \mathrm{L}^{-1}\right)$ were measured in the laboratory in accordance with the standards established by the American Public Health Association (APHA, 2005). We measured temperature $\left({ }^{\circ} \mathrm{C}\right.$ mercury thermometer), $\mathrm{pH}$ (DMPH pHmeter; Digimed, Santo Amaro, Brazil), depth (in $\mathrm{cm}$ ), transparency (Secchi disk) and electrical conductivity (in $\mu \mathrm{S} \mathrm{cm}^{-1}$, CD-28 conductometer; Digimed) on site. The rainfall data were obtained from the National Meteorology Institute - 8th Meteorology District, which has a weather station near the study area.

\section{Information Processing}

Multivariate species composition data on Trachelomonas and Strombomonas in relation to environmental factors were analyzed in two different ways. First, data related to the presence and absence of taxa, at different times of the year, were analyzed using cluster analysis. Subsequently, we applied indicator species analysis to the array of biological data, defining indicator species as those that showed significance on the Monte Carlo test $(\mathrm{p}<0.05)$.

Principal component analysis was applied to the physicochemical data matrix, in order to determine the significance of the accumulated variance of the explanatory environmental variables in the ordination. Finally, to reveal the main gradients of change in the composition of the indicator species, in relation to environmental processes, we applied canonical correspondence analysis (Ter Braak, 1986). The physical and chemical data were logarithmically transformed $(\log \mathrm{x})$, and analyses were processed using the program PC-ORD (McCune and Mefford, 1999).

\section{Results and discussion}

\section{Taxonomic composition}

We identified specimens of the genus Trachelomonas in all 24 of the samples analyzed, whereas we identified Strombomonas in only nine. Overall, we identified 22 taxa at the specific and infraspecific level, 20 Trachelomonas species and 2 Strombomonas species, distributed among 14 typical varieties and eight atypical varieties.

\section{Taxonomic survey}

Division: Euglenophyta

Class: Euglenophyceae

Order: Euglenales

Family: Euglenaceae

Genus: Trachelomonas Ehrenberg emend. Deflandre 1926.

1. Trachelomonas armata (Ehrenberg) Stein var. steinii Lemmermann emend. Deflandre, Abh. Naturw. Ver. Bremen 18:165. 1905.

Fig. 1 
Table 1. Details of samples collected from the Lago da Ponte, within the Porto Alegre Botanical Garden, and deposited in the Dr. Alarich R. H. Schultz Herbarium (HAS), in the city of Porto Alegre, state of Rio Grande do Sul, Brazil.

\begin{tabular}{|c|c|c|c|c|}
\hline Accession no. & Collecting Station & Collectors & Dates & Season \\
\hline 107564 & 1 & Alves-da-Silva, S. M., Friedrich, F. \& Nunes, M. L. & $25 \mathrm{Jul} 2007$ & Winter \\
\hline 107565 & 2 & Alves-da-Silva, S. M., Friedrich, F. \& Nunes, M. L. & $25 \mathrm{Jul} 2007$ & Winter \\
\hline 107566 & 1 & Alves-da-Silva, S. M. \& Friedrich, F. & 15 Aug 2007 & Winter \\
\hline 107567 & 2 & Alves-da-Silva, S. M. \& Friedrich, F. & 15 Aug 2007 & Winter \\
\hline 107568 & 1 & Friedrich, F. \& Nunes, M. L. & 12 Sep 2007 & Winter \\
\hline 107569 & 2 & Friedrich, F. \& Nunes, M. L. & 12 Sep 2007 & Winter \\
\hline 107570 & 1 & Friedrich, F. \& Nunes, M. L. & 10 Oct 2007 & Spring \\
\hline 107571 & 2 & Friedrich, F. \& Nunes, M. L. & 10 Oct 2007 & Spring \\
\hline 107572 & 1 & Alves-da-Silva, S. M. \& Friedrich, F. & 19 Nov 2007 & Spring \\
\hline 107573 & 2 & Alves-da-Silva, S. M. \& Friedrich, F. & 19 Nov 2007 & Spring \\
\hline 107574 & 1 & Alves-da-Silva, S. M. \& Friedrich, F. & 13 Dec 2007 & Spring \\
\hline 107575 & 2 & Alves-da-Silva, S. M. \& Friedrich, F. & 13 Dec 2007 & Spring \\
\hline 107576 & 1 & Alves-da-Silva, S. M., Nunes, M. L. \& Cunha, G.R. & 09 Jan 2008 & Summer \\
\hline 107577 & 2 & Alves-da-Silva, S. M., Nunes, M. L. \& Cunha, G.R. & 09 Jan 2008 & Summer \\
\hline 107794 & 1 & Alves-da-Silva, S. M., Friedrich, F. \& Nunes, M. L. & 13 Feb 2008 & Summer \\
\hline 107797 & 2 & Alves-da-Silva, S. M., Friedrich, F. \& Nunes, M. L. & 13 Feb 2008 & Summer \\
\hline 107800 & 1 & Friedrich, F. \& Nunes, M. L. & 14 Mar 2008 & Summer \\
\hline 107803 & 2 & Friedrich, F. \& Nunes, M. L. & 14 Mar 2008 & Summer \\
\hline 107806 & 1 & Friedrich, F. \& Nunes, M. L. & 11 Apr 2008 & Autumn \\
\hline 107809 & 2 & Friedrich, F. \& Nunes, M. L. & 11 Apr 2008 & Autumn \\
\hline 107812 & 1 & Friedrich, F. \& Nunes, M. L. & 13 May 2008 & Autumn \\
\hline 107815 & 2 & Friedrich, F. \& Nunes, M. L. & 13 May 2008 & Autumn \\
\hline 107818 & 1 & Alves-da-Silva, S. M. \& Friedrich, F. & 13 Jun 2008 & Autumn \\
\hline 107821 & 2 & Alves-da-Silva, S. M. \& Friedrich, F. & 13 Jun 2008 & Autumn \\
\hline
\end{tabular}

Lorica ovate, 32-37 $\mu \mathrm{m}$ long; $22-23 \mu \mathrm{m}$ wide; $\mathrm{Rc} / \mathrm{l}=1.4$, rounded poles; flagellar pore ca. $3 \mu \mathrm{m}$ diam., ring thickening present, collar absent; wall reddish-brown medium to dark, punctuate, smaller spinules on the anterior pole, larger curved converging spinules on the posterior pole; numerous chloroplasts, discoid, ca. $3.5 \mu \mathrm{m}$ diam.; internal pyrenoids present. Trachelomonas armata differs from the typical species by its smaller spinules on the anterior pole and larger converging spinules on the posterior pole.

Material examined: HAS107570, HAS107572, HAS107573, HAS107574, HAS107575, HAS107576, HAS107797, HAS107800.

Geographic distribution in Brazil, by region (state or district): Central-West (Mato Grosso, Federal District), North (Amazonas, Acre), Southeast (Espírito Santo, São Paulo, Rio de Janeiro), South (Paraná, Santa Catarina, Rio Grande do Sul).

2. Trachelomonas curta Cunha emend. Deflandre var. curta, Bull. Soc bot., 44: 286. 1927.

Fig. 2
Lorica subspherical, smooth, 18-20 $\mu \mathrm{m}$ long; $23-24 \mu \mathrm{m}$ wide; $\mathrm{Rc} / \mathrm{l}=0.7-0.9$; anterior-posterior flattening of the poles; flagellar pore ca. $2.5 \mu \mathrm{m}$ diam., annular thickening present, collar absent, yellow-brown wall; more than 10 chloroplasts, discoid, $4 \mu \mathrm{m}$ diam., numerous rod-shaped paramylon granules, ca. $2 \mu \mathrm{m}$ length.

Material examined: HAS107566, HAS107571, HAS107572, HAS107577, HAS107797, HAS107806, HAS107809, HAS107812.

Geographic distribution in Brazil, by region (state or district): Central-West (Federal District), North (Amazonas, Acre, Rondônia), Southeast (Minas Gerais, São Paulo, Rio de Janeiro), South (Paraná, Rio Grande do Sul).

3. Trachelomonas curta Cunha emend. Deflandre var. minima Tell \& Zalocar, Nova Hedwigia, 1: 364, pl. 8, fig. 21a-b, 22, pl. 14, fig. 3. 1985.

Fig. 3-4

Lorica subspherical, smooth, 4.5-8 $\mu \mathrm{m}$ long; 6-12.6 $\mu \mathrm{m}$ wide; $\mathrm{Rc} / \mathrm{l}=0.6-0.7$; anterior-posterior flattening of the poles. Trachelomonas curta differs from the typical species by its smaller dimensions. 
Material examined: HAS107564, HAS107566, HAS107567, HAS107569, HAS107570, HAS107571, HAS107572, HAS107573, HAS107574, HAS107575, HAS107576, HAS107577, HAS107806, HAS107809.

Geographic distribution in Brazil, by region (state): North (Amazon), Southeast (São Paulo), South (Paraná, Rio Grande do Sul).

4. Trachelomonas elliptica (Playfair) Deflandre, Bull. Soc Bot. France 74: 286. 1927.

Fig. 5-6

Lorica elliptical, 24-26 $\mu$ mlong, $10-11.5 \mu \mathrm{m}$ wide; $\mathrm{Rl} / \mathrm{c}=2.3$, slightly narrowed anterior pole; rounded posterior pole; flagellar pore ca. $2.5 \mu \mathrm{m}$ diam., high collar, $3 \mu \mathrm{m}$ high by $3 \mu \mathrm{m}$ wide; wall smooth, reddish-brown; numerous chloroplasts, discoid, ca. $2 \mu \mathrm{m}$ diameter.

Material examined: HAS107568, HAS107573, HAS107574, HAS107575, HAS107576.

Geographic distribution in Brazil, by region (state): Central-West (Mato Grosso), South (Rio Grande do Sul).

5. Trachelomonas gracillima Balech \& Dastugue var. gracillima, Physis 12: 355, fig. 2: 7. 1938.

Fig. 7

Lorica elliptical, 40-42 $\mu \mathrm{m}$ long, 17.0-17.5 $\mu \mathrm{m}$ wide; $\mathrm{Rl} / \mathrm{c}=2.4$; parallel sides, acuminate posterior pole, with three larger conical spinules in the posterior region, the others regularly distributed throughout the lorica; wall punctuated, reddish-brown; numerous chloroplasts, discoid, ca. $1.8 \mu \mathrm{m}$ diam.; internal pyrenoids present.

Material examined: HAS107571, HAS107572, HAS107574, HAS107575.

Geographic distribution in Brazil, by region (state): Central-West (Mato Grosso do Sul), South (Rio Grande do Sul).

6. Trachelomonas hemisphaerica Garcia de Emiliani, Rev. Asoc. Cienc. Nat. Litoral, 14(2): 241. 1983.

Fig. 8-9

Lorica hemispherical, $15-18 \mu \mathrm{m}$ long, $22-28 \mu \mathrm{m}$ in wide; $\mathrm{Rl} / \mathrm{c}=0.6$ to 0.7 ; rounded apical view; anterior and posterior pole separated by a raised edge, rounded posterior pole and anterior hemispheric slightly convex; apical median pore, conspicuous; smooth wall, russet; numerous chloroplasts, discoid, ca. $1.8 \mu \mathrm{m}$ diameter.

Material examined: HAS107809, HAS107812.

Geographic distribution in Brazil, by region (state): Central-West (Mato Grosso, Goiás), Southeast (Rio de Janeiro), South (Rio Grande do Sul, Paraná).

7. Trachelomonas hispida (Perty) Stein emend. Deflandre var. hispida, Revue gén. Bot., 38: 650, pl. 3, fig. 203, 207208. 1926.

Fig. 10-11
Lorica elliptical, 22-25 $\mu \mathrm{m}$ long, 17-20.5 $\mu \mathrm{m}$ wide; $\mathrm{Rl} / \mathrm{c}=1.2-1.3$; small conical spinules distributed throughout the lorica wall.

Material examined: HAS107570, HAS107571, HAS107572, HAS107573.

Geographic distribution in Brazil, by region (state or district): Central-West (Federal District, Mato Grosso, Mato Grosso do Sul, Goiás), North (Acre, Amazonas, Pará, Rondônia), Northeast (Pernambuco), Southeast (São Paulo, Rio de Janeiro), South (Paraná, Santa Catarina, Rio Grande do Sul).

8. Trachelomonas hispida (Perty) Stein emend. Deflandre var. coronata Lemmermann, in Pascher, Süsswasserfl. Deutsch. 2 (2): 150. 1913.

Fig. 12-13

Lorica elliptical, 35-41 $\mu \mathrm{m}$ long, 22-25 $\mu \mathrm{m}$ wide; $\mathrm{Rl} / \mathrm{c}=1.5-1.8$; rounded poles, flagellar pore ca. $3 \mu \mathrm{m}$ diam.; ring thickening present, collar ca. 3.5 to $4.5 \mathrm{high}, 7-9 \mu \mathrm{m}$ wide; punctuate wall, russet; conical spinules, distributed across the entire lorica surface, 1.6-1.8 $\mu \mathrm{m}$ long; chloroplasts, discoid, 3-4.5 $\mu \mathrm{m}$ diam.; diplopyrenoids present; numerous elliptical paramylon granules, ca. $2 \mu \mathrm{m}$ long; conical spinules distributed across the entire lorica surface.

Trachelomonas hispida differs from the typical species by its crown-shaped collar of spinules attached to its base (Fig. 13).

Material examined: HAS107566, HAS107569, HAS107571, HAS107573, HAS107574, HAS107577, HAS107794, HAS107797, HAS107800, HAS107803, HAS107815, HAS107818.

Geographic distribution in Brazil, by region (state or district): Central-West (Federal District, Goiás, Mato Grosso do Sul), North (Pará, Acre), Southeast (São Paulo), South (Rio Grande do Sul).

9. Trachelomonas lacustris Drezepolski var. lacustris, Kosmos, 50: 217, pl. 2, fig. 67. 1925.

Fig. 14

Lorica cylindrical or oblong, 25.5-36.5 $\mu \mathrm{m}$ long, $12-19 \mu \mathrm{m}$ wide; $\mathrm{Rl} / \mathrm{c}=1.9-2.1$; rounded poles, $2-3 \mu \mathrm{m}$ flagellar pore diam., ring thickening present; collar sometimes present; discoid chloroplasts; internal pyrenoids present; wall punctuate, russet; conical spinules, ca. $1 \mu \mathrm{m}$ long, regularly distributed across the surface of the lorica.

Material examined: HAS107564, HAS107569, HAS107570, HAS107571, HAS107572, HAS 107573 HAS107574, HAS107575, HAS107576, HAS107794, HAS107803, HAS107809.

Geographic distribution in Brazil, by region (state or district): Central-West (Federal District, Mato Grosso do Sul, Mato Grosso), North (Rondônia) Northeast (Pernambuco), Southeast (São Paulo, Rio de Janeiro), South (Rio Grande do Sul). 
10. Trachelomonas lemmermannii Woloszynska emend. Deflandre var. lemmermannii, Revué gen. Bot., 38:696, pl.7, fig.397, 380, 396-398, 405-406. 1926.

Fig. 15-16

Lorica conical-cylinder, 25-26 $\mu \mathrm{m}$ long, 14-15.5 $\mu \mathrm{m}$ wide; $\mathrm{Rl} / \mathrm{c}=1.7$; straight sides to the anterior $2 / 3$; anterior pole slightly truncated, posterior pole abruptly attenuated, tapered-rounded, flagellar pore ca. $4 \mu \mathrm{m}$ diam., ring thickening present, collar absent; wall punctuate, russet, conical spinules, irregularly distributed across the surface of the lorica, ca. $1 \mu \mathrm{m}$ long; discoid chloroplasts, ca. $3.5 \mu \mathrm{m}$ diameter. Material examined: HAS107573.

Geographic distribution in Brazil, by region (state or district): Central-West (Federal District, Mato Grosso do Sul, Mato Grosso, Goiás), Southeast (São Paulo, Rio de Janeiro), South (Paraná, Rio Grande do Sul).

11. Trachelomonas lemmermannii Woloszynska emend. Deflandre var. acuminata Deflandre, Revue gén. Bot., v.38, p.697, pl. 7, fig.404, 407-409,1926.

Fig. 17

Lorica conical-cylinder, 36-37 $\mu \mathrm{m}$ long, 15-16 $\mu \mathrm{m}$ wide; $\mathrm{Rl} / \mathrm{c}=2.3-2.4$; straight sides in the anterior and conical, tapered rounded posterior; flagellar pore ca. $4 \mu \mathrm{m}$ diam.; ring thickening present, collar absent; wall punctuate, light brown; discoid chloroplasts, ca. $3 \mu \mathrm{m}$ diam.; internal pyrenoids present.

Material examined: HAS107568, HAS107572, HAS107574, HAS107576, HAS107794, HAS107797, HAS107809.

Geographic distribution in Brazil, by region (state or district): Central-West (Mato Grosso, Federal District), Southeast (Rio de Janeiro), South (Rio Grande do Sul).

12. Trachelomonas oblonga Lemmermann var. truncata Lemmermann, Abh. Naturw. Ver. Bremen, 16: 344, 1899.

Fig. 18-20

Loricaoblong, 11-14 $\mu$ mlong, $10-11 \mu$ m wide; $\mathrm{Rl} / \mathrm{c}=1.1-1.2$, truncated anterior pole; rounded posterior pole, flagellar pore ca. $2 \mu \mathrm{m}$ diam., ring thickening present, collar absent; wall smooth, light brown to medium reddish-brown.

Material examined: HAS107569, HAS107570, HAS107572, HAS107573, HAS107574, HAS107575, HAS107576, HAS107577.

Geographic distribution in Brazil, by region (state): Central-West (Goiás), North (Amazon), South (Santa Catarina, Rio Grande do Sul).

13. Trachelomonas pulcherrima Playfair var. pulcherrima, Proc. Linn. Soc. N. S. W., 40:13, pl. 1, figs.32-33, 1915.

Fig. 21

Lorica elliptical, $22-23 \mu \mathrm{m}$ long, $10-11 \mu \mathrm{m}$ wide; $\mathrm{Rl} / \mathrm{c}=$ 2.1-2.2; pole rounded to slightly truncated, rounded posterior pole, flagellar pore ca. $2 \mu \mathrm{m}$ diam., ring thickening pre- sent, collar absent; wall smooth or punctuate, light brown to medium reddish-brown; discoid chloroplasts, ca. 1.5 diam.; internal pyrenoids present.

Material examined HAS107572, HAS107573, HAS107574, HAS107576, HAS107577, HAS107809, HAS107818.

Geographic distribution in Brazil, by region (state): Central-West (Mato Grosso), North (Amazon), Southeast (São Paulo, Rio de Janeiro), South (Rio Grande do Sul).

14. Trachelomonas pulcherrima Playfair var. minor Playfair, Proc. Linn. Soc. N.S.W., 40:14, pl. 1, figs.37-38, 1915.

Fig. 22-24

Lorica elliptical, $14-15 \mu$ mlong, 7.5-9 $\mu$ m wide; Rl/c:1.7-1.9; pole rounded to slightly truncated, rounded posterior pole, flagellar pore ca. $2 \mu \mathrm{m}$ diam., ring thickening present, collar absent; wall punctuate, light russet; chloroplasts discoid, ca. 1.8 diam.; no internal pyrenoids observed.

Trachelomonas pulcherrima var. minor differs from the typical species by its smaller cell dimensions.

Material examined: HAS107573, HAS107574, HAS107575, HAS107576, HAS107794, HAS107797.

Geographic distribution in Brazil, by region (state): Southeast (Rio de Janeiro), South (Paraná, Rio Grande do Sul, Santa Catarina).

15. Trachelomonas robusta Swirenko emend. Deflandre var. robusta, Révue gén. Bot., 38: 657, pl. 4,fig. 257, 260. 1926. Fig. 25

Lorica broadly elliptical, $25-26 \mu \mathrm{m}$ long, 20-21 $\mu \mathrm{m}$ wide; $\mathrm{Rl} / \mathrm{c}=1.2$, rounded poles, flagellar pore ca. $2 \mu \mathrm{m}$ diam., ring thickening present; collar absent; wall russet, thickly scored, conical spinules, $\leq 2.5 \mu \mathrm{m}$ long, irregularly distributed across the wall surface; chloroplasts discoid, ca. $2 \mu \mathrm{m}$ diam.; internal pyrenoids present.

Material examined: HAS107567, HAS107570, HAS107572, HAS107576.

Geographic distribution in Brazil, by region (state or district): Central-West (Mato Grosso, Federal District), North (Pará, Amazonas), Southeast (São Paulo, Rio de Janeiro) South (Rio Grande do Sul).

16. Trachelomonas sculpta Balech var. sculpta, An. Mus. argent. Cienc. nat., 41: 245, fig. 32, 167. 1944.

Fig. 26-27

Lorica spherical, 20-21 $\mu \mathrm{m}$ diam., flagellar pore in the interior of one depression, ca. $2 \mu \mathrm{m}$ diam., ring thickening present; collar absent, thick wall, ca. $1.8 \mu \mathrm{m}$ diam.; wall dark brown, depressions approximately polygonal, isometric, dense, evenly distributed; no chloroplasts or pyrenoids observed.

Material examined: HAS107567, HAS107573.

Geographic distribution in Brazil, by region (state): Central-West (Mato Grosso do Sul), North (Amazon), Southeast (Rio de Janeiro), South (Santa Catarina, Paraná, Rio Grande do Sul). 


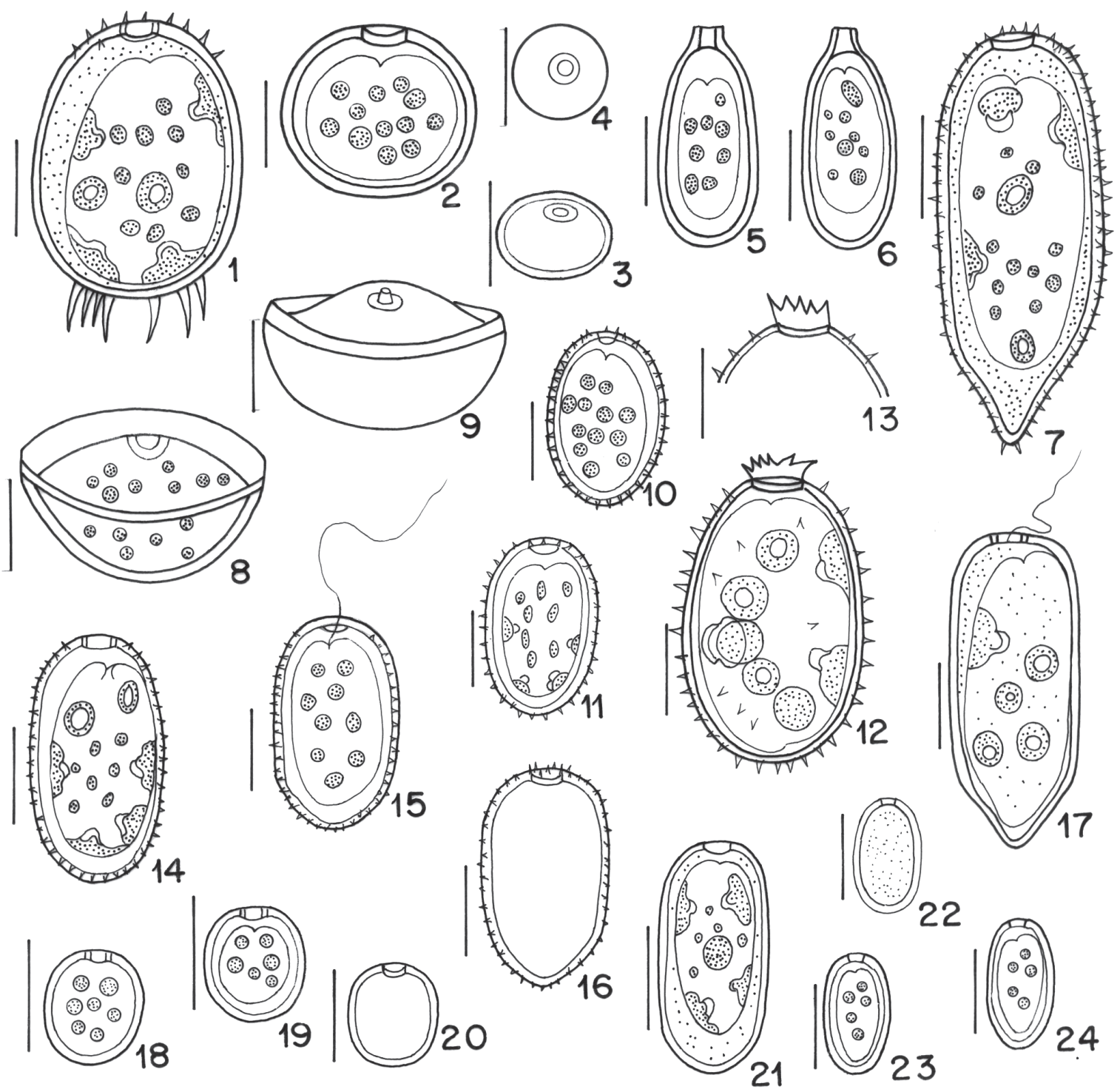

Figures 1-24. Trachelomonas from the Lago da Ponte. 1. Trachelomonas armata (Ehr.) Stein var. steinii Lemm. emend. Defl. 2. Trachelomonas curta Cunha emend. Defl. var. curta. 3, 4. Trachelomonas curta Cunha emend. Defl. var. minima Tell \& Zal. 5, 6. Trachelomonas elliptica (Playf.) Defl. 7. Trachelomonas gracillima Bal. \& Dast. var. gracillima. 8, 9. Trachelomonas hemisphaerica Garc. de Emil. 10, 11 Trachelomonas hispida (Perty) Stein emend. Defl. var. hispida. 12, 13. Trachelomonas hispida (Perty) Stein emend. Defl. var. coronata Lemm. 13. Detail of collar. 14. Trachelomonas lacustris Drez. var. lacustris. 15, 16. Trachelomonas lemmermannii Wolosz. emend. Defl. var. lemmermannii. 17. Trachelomonas lemmermannii Wolosz. emend. Defl. var. acuminata Defl. 18-20. T. oblonga Lemm. var. truncata Lemm. 21. Trachelomonas pulcherrima Playf. var. pulcherrima. 22-24. Trachelomonas pulcherrima Playf. var. minor Playf. 22. Detail of the wall with punctuations. Scale $=10 \mu \mathrm{m}$.

17. Trachelomonas varians Deflandre var. varians, Revue gén. Bot.,v.38,p.525, fig.37-40,42-44,47- 50,1926.

Fig. 28-30

Lorica globose, $24-25 \mu \mathrm{m}$ diam., ring thickening present; inner tube cylindrical, straight or slightly oblique, extended by a slightly oblique outer edge; wall smooth, dark brown, ca. $1.8 \mu \mathrm{m}$ thick; chloroplasts numerous, discoid.

The genera T. varians and T. cervicula Stokes are quite similar and difficult to distinguish by their descriptions. The individuals examined were identified in accordance with Rino \& Pereira (1991), who cite a short, truncated, conical, often oblique, inner tube extended by a flange that can be externally oblique, with numerous chloroplasts (30-50); lorica globose to broadly elliptical. Those same authors stated that T. cervicula possesses a spherical to subspherical lorica, has a long straight inner tube and presents 6-10 chloroplasts, all containing haplopyrenoids.

Material examined: HAS107573.

Geographic distribution in Brazil, by region (state or district): Central-West (Mato Grosso, Federal District), Northeast (Pernambuco), South (Paraná, Rio Grande do Sul). 
18. Trachelomonas volvocina Ehrenberg var. volvocina, Infusoria. 18, pl. 2, fig. 29. 1838.

Fig. 31

Lorica spherical, smooth, 17-20 $\mu \mathrm{m}$ diam.; flagellar pore ca. $3 \mu \mathrm{m}$ diam., ring thickening present; collar absent; wall reddish, smooth; two shield-shaped chloroplasts, with diplopyrenoids; no paramylon granules observed.

Material examined: HAS107567, HAS107573, HAS107574, HAS107575, HAS107576, HAS107577, HAS107794, HAS107797, HAS107803, HAS107806, HAS107809, HAS107812, HAS107815.

Geographic distribution in Brazil, by region (state): Central-West (Mato Grosso, Mato Grosso do Sul, Goiás), Northeast (Bahia, Pernambuco), North (Acre, Amazonas, Manaus, Rondônia), Southeast (Espírito Santo, Minas Gerais, Sao Paulo, Rio January), South (Paraná, Santa Catarina, Rio Grande do Sul).

19. Trachelomonas volvocina Ehrenberg var. derephora Conrad, Ann. Biol. Lacustre 8: 201, pl.1, fig. 3. 1916.

Fig. 32-33

Lorica spherical, 20-22 $\mu \mathrm{m}$ diam.; flagellar pore ca. $3 \mu \mathrm{m}$ diam., ring thickening present; collar cylindrical, $2 \mu \mathrm{m}$ long; wall reddish, smooth; chloroplasts numerous, discoid, ca. 2 $\mu \mathrm{m}$ diam., no internal pyrenoids observed.

Material examined: HAS107569, HAS107573, HAS107575. Geographic distribution in Brazil, by region (state): Central-West (Mato Grosso), North (Amazon), Southeast (São Paulo, Rio de Janeiro), South (Rio Grande do Sul).

20. Trachelomonas volvocinopsis Swirenko var. volvocinopsis, Arch. Hydrobiol. Planktonk., 9:33, pl. 19, figs. 1-3, 1914. Fig. 34

Lorica spherical, 21.0-25.5 $\mu \mathrm{m}$ diam.; flagellar pore ca. $2.5 \mu \mathrm{m}$ diam., ring thickening present; collar absent; wall russet, smooth; chloroplasts (>10), discoid, ca. $3 \mu \mathrm{m}$ diameter.

Material examined: HAS107564, HAS107565, HAS107567, HAS107568, HAS107569, HAS107570, HAS107571, HAS107572, HAS107573, HAS107574, HAS107575, HAS107576, HAS107577, HAS107794, HAS107797, HAS107800, HAS107803, HAS107806, HAS107809, HAS107812, HAS107815, HAS107818, HAS107821.

Geographic distribution in Brazil, by region (state or district): Central-West (Mato Grosso, Mato Grosso do Sul, Goias, Federal District), Northeast (Pernambuco, Rio Grande do Norte), North (Amazonas, Rondônia, Pará), Southeast (Minas Gerais, Sao Paulo, Rio de Janeiro), South (Santa Catarina, Paraná, Rio Grande do Sul).

Genus Strombomonas Deflandre 1930.

21. Strombomonas costata Deflandre var. costata, Arch. Protistenk. 69(3): 589, figs. 76-80. 1930.

Fig. 35-37
Lorica ovate to elliptical, sometimes rhomboid; $53-58 \mu \mathrm{m}$ long, 23-27 $\mu$ m wide, $\mathrm{Rc} / \mathrm{l}=2.2-2.3$; apical view, with rounded edge slightly lobed; anterior pole gradually attenuated in cylindrical collar, extended at the end, crenulated edge, 4-6.5 $\mu \mathrm{m}$ high and 6-7 $\mu \mathrm{m}$ wide; posterior pole abruptly attenuated in hyaline flow process, tapered, straight or slightly curved, 11-13 $\mu \mathrm{m}$ long; wall slightly roughened, hyaline to yellowish light brown, with longitudinal folds; numerous chloroplasts, ca. $3 \mu \mathrm{m}$ diam., flagellum ca. the length of the lorica.

Material examined: HAS107570, HAS107571, HAS107573, HAS107574.

Geographic distribution in Brazil, by region (state): North (Pará, Amazonas), Southeast (Rio de Janeiro), South (Santa Catarina, Rio Grande do Sul).

22. Strombomonas fluviatilis (Lemmermann) Deflandre var. levis (Lemmermann) Skvortzov, Aus der Biol. Sungari Station zur Harbin 1(2): 78, pl. 6, fig. 33. 1925.

Fig. 38-39

Lorica elliptical, 64-70.5 $\mu \mathrm{m}$ long, 26-30 $\mu \mathrm{m}$ wide, Rc/l $=2.1-2.5$; anterior pole ending in short collar, broad at the distal region, with 4-6 $\mu \mathrm{m}$ high and 6-7 $\mu \mathrm{m}$ wide; posterior pole ending in straight caudal process, tapered, 14-17 $\mu \mathrm{m}$ long; rough wall, hyaline to greenish, with or without internal pyrenoids (8-10); chloroplasts numerous, discoid, 3-5 $\mu$ m diameter.

Material examined: HAS107569, HAS107573, HAS107576, HAS107797, HAS107812, HAS107815.

Geographic distribution in Brazil, by region (state): Central-West (Mato Grosso), North (Amazon), Southeast (Rio de Janeiro), South (Rio Grande do Sul).

\section{Geographic distribution}

Of the 22 taxa identified in the lake, $54.5 \%$ have a cosmopolitan distribution, whereas the others have been recorded on two to three continents. Trachelomonas armata var. steinii, T. hispida var. hispida, T volvocina var. volvocina and T. volvocinopsis var. volvocinopsis are taxa with broader distribution in Brazil.

All species and infraspecies had already been cited in Brazil, although T. elliptica and T. gracillima represented new records for Rio Grande do Sul.

The most representative taxa for their occurrence in the urban lake studied were Trachelomonas curta var. minima, T. hispida var. coronata, T. volvocina var. volvocina and $T$. volvocinopsis, the last taxon being distinctive in that it was present in $96 \%$ of the analyzed samples. With the exception of T. curta var. minima, the other species are distributed worldwide. The least representative taxa in the lake were T. hemisphaerica, T. lemmermannii var. lemmermannii, T. sculpta var. sculpta, T. varians var. varians and T. volvocina var. derephora, all of which occurred in less than $11 \%$ of the samples. 


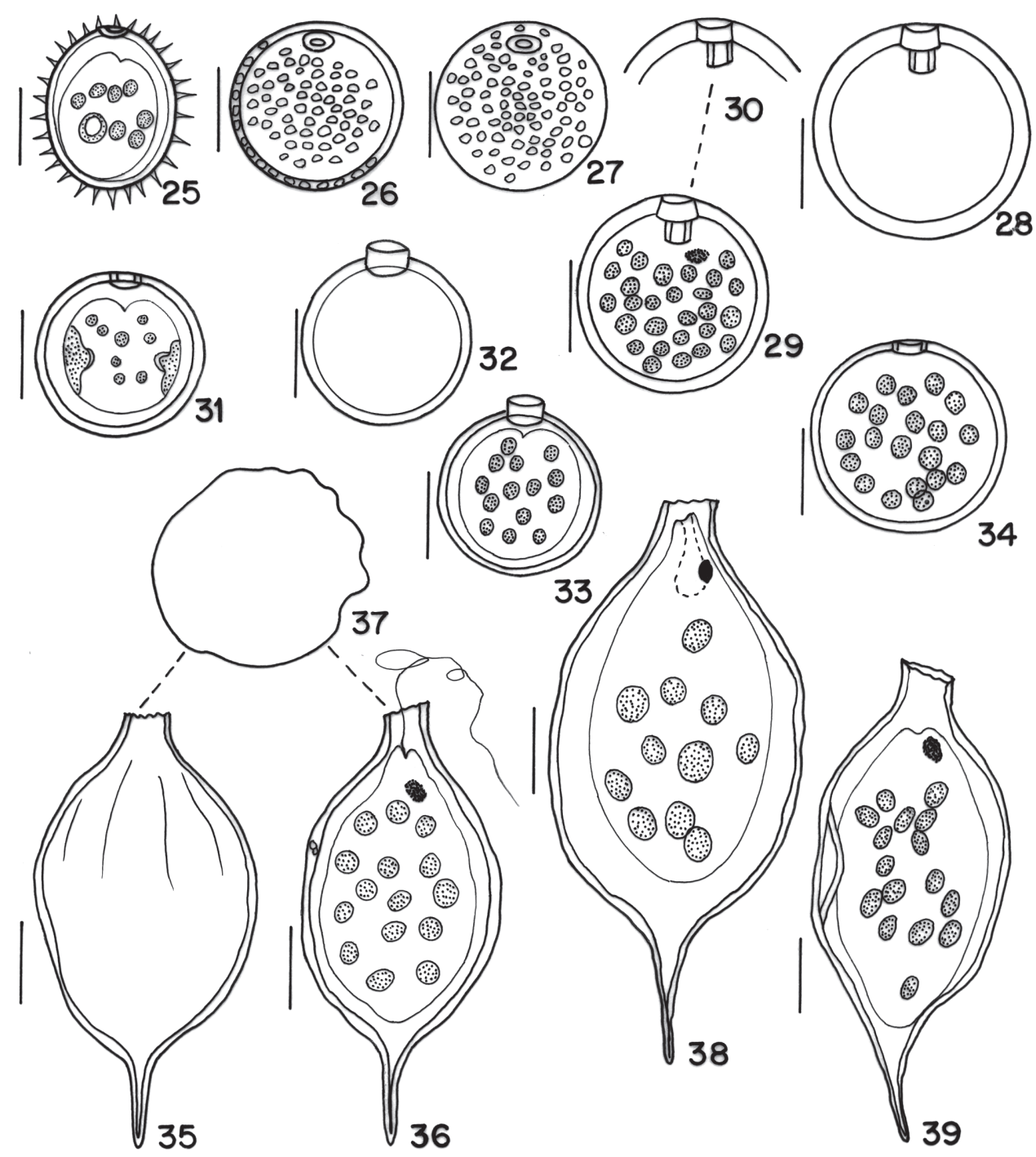

Figures 25-39. Trachelomonas and Strombomonas from the Lago da Ponte. 25. Trachelomonas robusta Swir. emend. Defl. var. robusta. 26, 27. Trachelomonas sculpta Bal. var. sculpta. 28-30. Trachelomonas varians Defl. var. varians. 30. detail of the inner collar. 31. Trachelomonas volvocina Ehr. var. volvocina. 32, 33. Trachelomonas volvocina Ehr. var. derephora Conr. 34. Trachelomonas volvocinopsis Swir. var. volvocinopsis. 35-37. Strombomonas costata Defl. var. costata. 37. apical view. $38,39$. Strombomonas fluviatilis (Lemm .) Defl. var. levis (Lemm.) Skv. Scale $=10 \mu \mathrm{m}$.

\section{Species richness}

The highest number of taxa was recorded in the spring of 2007 (Fig. 40). The richness ranged from one to 17 taxa, peaking in November 2007, when 17 taxa were cited at Station 2, corresponding to $73 \%$ of the taxa recorded.

\section{Cluster analysis}

Cluster analysis, based on data regarding the presence and absence of Trachelomonas and Strombomonas, as well as the categorical variable "seasonality" (in function of the different times of year), revealed three main clusters: the first comprising Winter samplings; the second comprising Spring samplings; and the third comprising samplings from
Summer and Fall (Fig. 41). The analysis revealed seasonal differences in the richness of the two genera.

\section{Indicator species analysis}

Figure 42 shows the species that showed significance $(\mathrm{p}<0.05)$ on a Monte Carlo test, having been selected for additional tests using canonical correspondence analysis in order to reveal the main gradients of change in species composition. The indicator species Trachelomonas armata var. steinii, T. curta var. minima, T. gracillima, T. hispida var. hispida, T. lacustris, T. oblonga var. truncata and Strombomonas costata form the Spring subcluster, corresponding to the Spring of 2007 and showing a significant seasonal component grouping. 


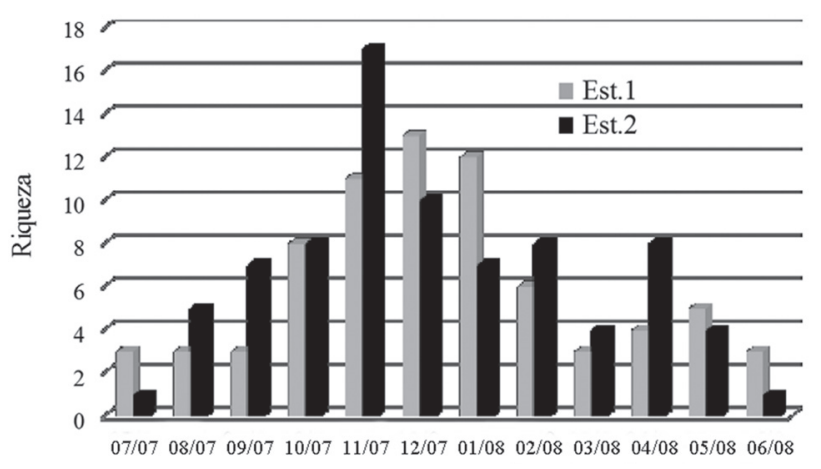

Figure 40. Distribution of the richness (no. de taxa) of Trachelomonas and Strombomonas in the Lago da Ponte, in the state of Rio Grande do Sul, Brazil, the two sampling stations (ST1= Station 1 and ST2= Station 2 ), between July 2007 and June 2008.

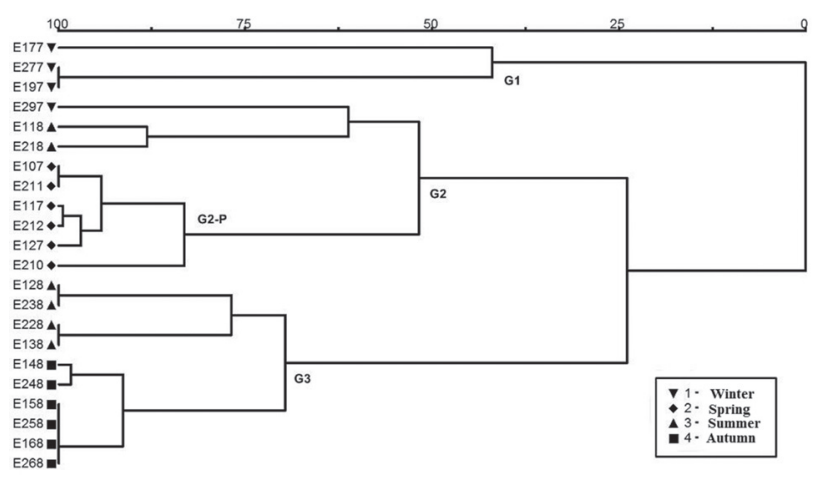

Figure 41. Cluster analysis based on monthly data related to the presence and absence of Trachelomonas and Strombomonas species and physicochemical variables for the Lago da Ponte, in the state of Rio Grande do Sul, Brazil, with seasonality as the categorical variable. G1 - Winter cluster; G2 - Spring cluster; G2-P - Spring subcluster; G3 - Summer/Autumn cluster.

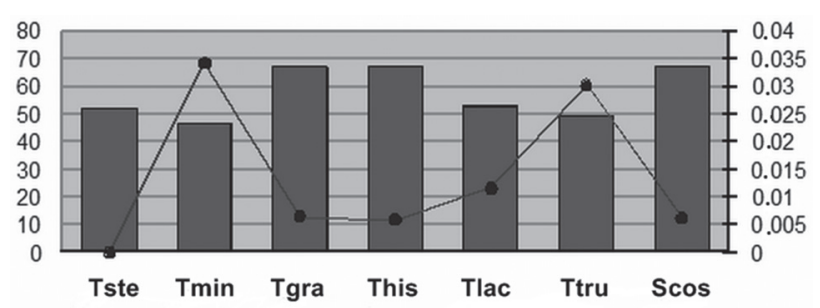

Indicative value $\rightarrow-$ Monte Carlo test

Figure 42. Indicator species analysis based on presence and absence data of Trachelomonas and Strombomonas at two stations (ST1 and ST2) in the Lago da Ponte, in the state of Rio Grande do Sul, Brazil, between July 2007 and June 2008. Tste - Trachelomonas armata var. steinii; Tmin - T. curta var. minima; Tgra - T. gracillima; This - T. hispida var. hispida; Tlac - T. lacustris; Ttru - T. oblonga var. truncata; Scos - Strombomonas costata.

\section{Principal component analysis}

The principal component analysis revealed that the first eight physical and chemical variables reached a cumulative percentage of variance of the explanatory order correspon- ding to the analysis cut-off point, $95 \%$ according to the variable selection methodology described by Delevati et al. (2005). The other two variables whose cumulative percentage of variance exceeded the cut-off were excluded from a second analysis to avoid the problem of multicollinearity between environmental variables, particularly in the case of multivariate analysis (Ter Braak, 1986).

\section{Ordination: canonical correspondence analysis}

The canonical correspondence analysis revealed 58.7\% of the total variability of the data on its first 3 axes, with eigenvalues for axes 1,2 and 3 of $41.6,10.0$ and 7.2, respectively. This relatively low explicability, however, is expected in the ordination analysis of ecological data, confirming the complexity of factors acting in determining the composition of ecosystems (Ter Braak and Prentice, 1988). However, the species-environment correlations for axes 1,2 and 3 $(r=0.939, r=0.847$ and $r=0.780$, respectively) indicated a strong relationship between species distribution and the environmental variables used in the ordination. The Monte Carlo permutation test revealed that sequence axis 1 was statistically significant $(\mathrm{p}<0.05)$, indicating a high likelihood of not being a result of chance.

With respect to canonical correspondence analysis axis 1 (which explained $41.6 \%$ of the variance), the most important environmental variable in the sequence was, in conformity with the intra-set correlation coefficients, ammonia $(r=0.581)$, which correlated with the positive quadrant of axis 1 (Fig. 43), revealing an environmental gradient for this nutrient, which ranged from $33.2 \pm 38.8 \mu \mathrm{g} \mathrm{L}^{-1}$ in Winter to $56.7 \pm 12.1 \mu \mathrm{g} \mathrm{L}{ }^{-1}$ in the Spring, highlighting that the $\mathrm{pH}$ remained relatively acid throughout the year, with an average value of $6.0 \pm 0.05$ (coefficient of variation $=0.8 \%$ ). The ammonia concentrations observed, however, are relatively low in comparison with those reported by Baumgarten et al. (2003), who, working in the Lagoa dos Patos, found that ammonia concentrations ranged from 240 to $300 \mu \mathrm{g} \mathrm{L}{ }^{-1}$ in areas without anthropogenic disturbance. It is of note that values as low as those observed in the present study were reported by Nunes et al. (2006) for the Baia dos Coqueiros, in the Pantanal region along the coast of Brazil. Those authors reported the presence of macrophytes and found that the mean $24 \mathrm{~h}$ ammonia concentration was $33.0 \mu \mathrm{g} \mathrm{L}^{-1}$, with a $\mathrm{pH}$ of 5.4-7.0.

The ammonium ion is recognized as a good marker of recent urban pollution, mainly from domestic sewage (Pereira \& Mercante, 2005). Therefore, the low values observed in the present study could be explained by the fact that the lake evaluated is an artificial aquatic environment located within a protected area, the nitrogen content coming from rain, organic/inorganic material of allochthonous origin or fixation of molecular nitrogen within the lake itself. Because the $\mathrm{pH}$ of the lake is low (annual average of $6.0 \pm 0.05$ ), the ammonia formed is unstable and is converted by hydration to the ammonium ion, which becomes predominant in this 


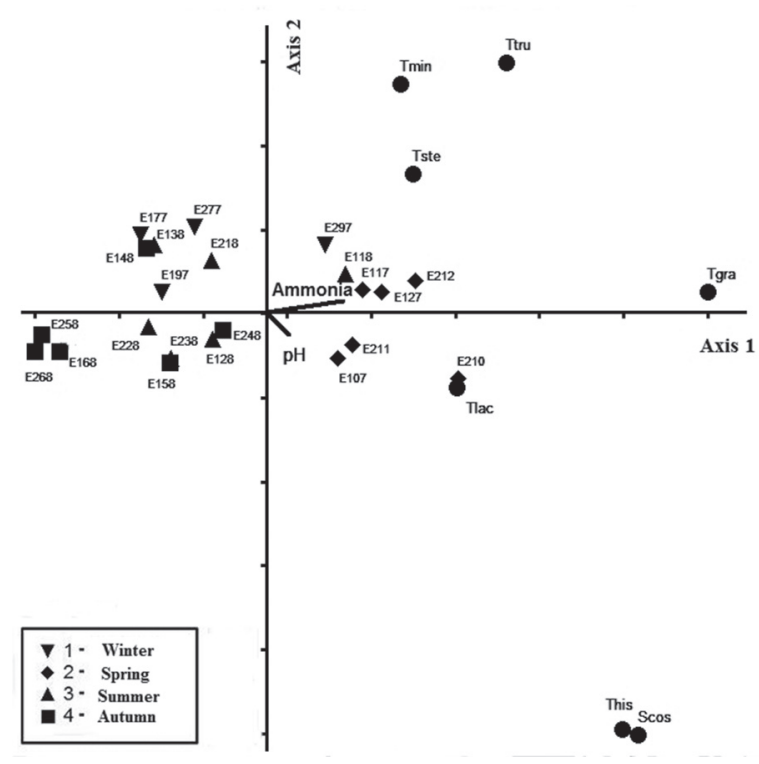

Figure 43. Scatter diagram based on canonical correspondence analysis considering indicator species and the physicochemical matrix of principal dispersion components for the Lago da Ponte, in the state of Rio Grange do Sul, Brazil, collected monthly from July 2007 to June 2008, with seasonality as the categorical variable.

Tste - Trachelomonas armata var. steinii; Tmin - T. curta var. minima; Tgra - T. gracillima; This - T. hispida var. hispida; Tlac - T. lacustris; Ttru - T. oblonga var. truncata; Scos - Strombomonas costata.

condition (Esteves, 1998; Pereira \& Mercante, 2005). We also found that, along the ammonia gradient, there was a clear differentiation of species that comprised the Spring subcluster, which were characterized as indicator species (Fig. 42). There is therefore a high probability of there being species tolerant of low $\mathrm{pH}$ (acidic water, $\mathrm{pH}<7.0$ ), given that this condition was essential for the prevalence of the ammonium ion in the gradient detected, even at low concentrations, characterizing a natural environment. Alves-da-Silva \& Avila (1995) recorded 22 taxa of Trachelomonas in shallow lakes of the Zoological Garden and the Botanical Garden in the city of Porto Alegre, and 17 of those taxa were also identified in the present study. Those authors stated that species richness was greatest when the $\mathrm{pH}$ was acidic to neutral.

Alves-da-Silva \& Torres (1994b), Alves-da-Silva \& Bridi (2004) and Alves-da-Silva \& Bicudo, (2006) noted that genus Strombomonas tolerates a wide range of $\mathrm{pH}$ values, occurring in slightly acidic to alkaline waters. In this context, the results of the present study corroborate those obtained by other researchers: Trachelomonas and Strombomonas achieve their greatest richness in water that is slightly acidic to slightly alkaline. These data increasing the ecological knowledge of this group of algae.

\section{Acknowledgments}

This study received financial support from the Brazilian Conselho Nacional de Desenvolvimento Científico e Tecno- lógico (CNPq, National Council for Scientific and Technological Development; Programa Institucional de Bolsas de Iniciação Científica [PIBIC, Institutional Program for Scientific Initiation Scholarships] Grant no. 101496/20109 to JCC). The authors would like to thank Manoel Luis Nunes, chemist in the Cryptogamic Botany Section, at the Museum of Natural Science of the Zoobotanical Foundation of the state of Rio Grande do Sul, for assisting in the sample collection and chemical analyses. We are also grateful to Rejane Rosa for the finished artwork.

\section{References}

Alves-da-Silva, S.M. \& Ávila, I. R. 1995. O gênero Trachelomonas Ehr. emend. Defl. (Euglenaceae) do Parque Zoológico de Sapucaia do Sul e do Jardim Botânico de Porto Alegre, Rio Grande do Sul, Brasil. Iheringia, Série Botânica 46: 27-56.

Alves-da-Silva, S.M. \& Bicudo, C. E. M. 2006. Contribution to the knowledge of genera Strombomonas (Euglenophyceae) of the state Rio Grande do Sul, southern Brazil. Algological Studies 119: 29-58.

Alves-da-Silva, S.M. \& Bridi, F. C. 2004. Euglenophyta no Parque Estadual Delta do Jacuí, Rio Grande do Sul, Brasil. 3. O gênero Strombomonas Defl. Acta Botanica Brasilica 18 (3): 555-571.

Alves-da-Silva, S. M. \& Torres, J. R. 1992. Estudo taxonômico do gênero Lepocinclis Perty (Família Euglenaceae), Parque Zoológico de Sapucaia do Sul e Jardim Botânico de Porto Alegre, Rio Grande do Sul, Brasil. Iheringia, Série Botânica 42: 87-104.

Alves-da-Silva, S. M. \& Torres, J. R. 1994a. O gênero Euglena Ehr. de sistemas lênticos do Parque Zoológico e Jardim Botânico, Rio Grande do Sul, BR. Revista Brasileira de Biologia 54(2): 345-363.

Alves-da-Silva, S.M. \& Torres, J.R. 1994b. O gênero Strombomonas (Euglenaceae) no Parque Zoológico, Sapucaia do Sul e do Jardim Botânico de Porto Alegre, Rio Grande do Sul, Brasil. Iheringia, Série Botânica 44: 15-32.

Alves-da-Silva, S.M. \& Torres, J.R. 1994c. Estudo taxonômico do gênero Phacus Duj. (Euglenaceae) no Parque Zoológico, Sapucaia do Sul e no Jardim Botânico, Porto Alegre Rio Grande do Sul. Iheringia, Série Botânica 44: 45-83.

APHA. 2005. American Public Health Association. Standard Methods for the Examination of water and Wastewater. 21 ed. APHA-AWWAWPCF, Washington D.C.

Baumgartem, M.G.; Niencheski, L.F. \& Veeck, L. 2003. Nutrientes na coluna da água e na água intersticial de sedimentos de uma enseada rasa estuarina com aporte de origem antrópica. Atlântica 123: 101-116.

Bourrelly, P.C. 1970. Les algues d'eau douce: initiation à la systématique. Tome III. Les algues blues et rouges. Les Eugléniens, Peridiniens et Cryptomonadines. Éditions N. Boubée, Paris, 3: 1- 512.

Branco, C.W.C. \& Senna, P.A.C. 1996. Phytoplankton composition, community structure and seasonal changes in a tropical reservoir (Paranoá Reservoir, Brazil). Algological Studies 81: 69-84.

Cecy, I.T. 1990. A Restinga do Pontal do Sul, município de Paranaguá, Pr. I- Levantamento ficológico (Euglenophyta) e físico-químico. Arquivos de biologia e tecnolologia 33(1): 1-79.

Cecy, I.T. 1986 Estudo das algas microscópicas (Nostocophyta, Euglenophyta, Chrysophyta e Chlorophyta) do Lago do Parque Barigüi, em Curitiba, Estado do Paraná, Brasil. Arquivos de biologia e tecnolologia 29(2): 383.

Cecy, I. T.; Moreira, I. M. V. \& Hohmann, E. 1976. Estudo ficológico e químico-bacteriológico da água do tanque do Passeio Público de Curitiba, Estado do Paraná, Brasil, 1: gêneros de algas microscópicas. Boletim do Museu Botânico Municipal 25: 1-37.

Camargo, J.C. \& Velho, L.F.M. 2010. Composition and species richness of flagellate protozoa from environments associated to the Baía river (Mato Grosso do Sul State, Brazil): influence of the hydrological period and the connectivity. Acta Scientiarum Biological Sciences 4: 349-356. 
Delevati, D. M.; Vaz, V. B.; Flesch, L. A. \& Preussler, M. F. 2005. O plano de bacia do Pardo: um relato de experiência. In: II Seminário Estadual e V Seminário Regional de Educação Ambiental, 2005. Anais... Santa Cruz do Sul, RS, Brasil, p. 21-37.

Domingues. C.D. \& Torgan, L.C. 2011. Fitoplâncton (exceto Chlorophyceae) de um lago artificial urbano no Sul do Brasil. Revista Brasileira de Botânica 34(3): 463-480.

Esteves, F.A. 1998. Fundamentos de Limnologia. Rio de Janeiro: Interciência. 2 ed., 602p.

Ferragut, C.; Lopes, M.R.M.; Bicudo, D.C.; Bicudo, C.E.M. \& Vercellino, I. S. 2005. Ficoflórula perifítica e planctônica (Exceto Bacillariophyceae) de um reservatório oligotrófico raso (Lagoa do IAG, São Paulo). Hoehnea 32(2): 137-184.

Ferreira, A.C.S. \& Menezes, M. 2000. Flora planctônica de um reservatório eutrófico. Lagoa Guandu, município de Nova Iguaçu. RJ. Hoehnea 27: 45-76

Franceschini, I.M. 1992. Algues d'eau douce de Porto Alegre Brésil (les Diatomophycées exclues). Bibliotheca Phycologica 92: 1-81.

García-Gil, L. J. \& Camacho, A. 2001. Anaerolimnología. Pequeña guía para el muestreo en aguas microaeróbicas y anóxicas en lagos y embalses estratificados. Limnetica 20: 179-186.

Goulart, E.; Couté, A.; Thérézian, Y. \& Francischini, I.M. 2002. Phytoplankton of lentic water from the Campus of Santa Catarina University (Florianópolis, SC, Southern Brazil). Ciência e Natura 24: 21-48.

Giani A.; Figueiredo C.C. \& Eterovick, P.C. 1999. Algas planctônicas do reservatório da Pampulha (MG): Euglenophyta, Chrysophyta, Pyrrophyta, Cyanobacteria. Revista Brasileira de Botânica 22: 107-116.

Heckaman, C.W.; Hardoim, E.I.; Ferreira, S.A. \& Kretzachmar, A.U. 1993. Preliminary observations on some cosmopolitan algae in ephemeral water bodies of the Pantanal, Mato Grosso, Brazil. National Institute of Ecology 279-292.

Huber-Pestalozzi, G. 1955. Euglenophyceen. Pp. 1-605. In: G. HuberPestalozzi (ed.). Das phytoplankton des Susswässers: Systematik und Biologie. Teil 4, E. Schweizerbart'sche Verlangsbuchhandlug, Stuttgart.

Huszar, V.L.M.; Silva, L.H.S. \& Esteves, F.A. 1990. Estrutura das comunidades fitoplântonicas de 18 lagoas da região do Baixo Rio Doce, Linhares, Espírito Santo, Brasil. Revista Brasileira de Biologia 50: 585-598.

Jati, S. \& Train, S. 1994. Euglenaceae pigmentadas de ambientes lênticos da Ilha Porto Rico, Município de Porto Rico, Paraná, Brasil. Iheringia, Série Botânica 45: 117-142.

Keppeler, E.C.; Lopes, M.R.M. \& Lima, S.M. 2002. Ficoflórula do Lago do Amapá em Rio Branco- Acre, I: Euglenophyceae. Revista Brasileira de Biologia 59(4): 679-686.

Leedale, G. Euglenoid Flagellates. Englewood: Clifs, NJ., Prentice Hall, 1967. 242p., il.

Martins, D. V.; Santana, C. L. \& Oliveira, O. C., 1991, Estudo qualitativo do fitoplâncton do Dique do Tororó, Salvador, Bahia, Brasil. Revista Brasileira de Biologia 51(2): 445-453.
Mccune, B. \& Mefford, M. J. 1999. PC-ORD. Multivariate Analysis of Ecological Data, Version 4. MjM Software Design, Gleneden Beach, Oregon, USA. 237p.

Németh, J. 1980. Az ostoros Algák (Euglenophyta). Budapeste: Vizdock. Vol. 1, 294p. (Série Hidrobiologia no 8).

Nogueira, I. S. \& Rodrigues, L. N. C. 1999. Algas planctônicas de um lago artificial do Jardim Botânico Chico Mendes, Goiânia, Goiás: floristica e algumas considerações ecológicas. Revista brasileira de Biologia 59(3): 377-395.

Nogueira, I.S.; Nabout, J. C.; Oliveira, J.E \& Silva, K.D. 2008. Diversidade (alfa, beta e gama) da comunidade fitoplanctônica de quatro lagos artificiais urbanos do município de Goiânia, GO. Hoehnea 35(2): 219-233.

Nunes, J. R.; Loverde, S.; Pinto-Silva, V.; Ferraz, L.; Amorin, A.; Costa, C. P.; Mondin, L. A.; Siveira, J. S.; Favalessa, O.; Gonçalvez, C. H.; Troy, W. P. \& Uriquiza, N. G. 2006. Variação diária das características limnológicas da baía dos Coqueiros, Pantanal de Poconé, MT. UNICiências 10: 31-46.

Pereira, L.P. \& Mercante, C.T. 2005. A amônia nos sistemas de criação de peixes e seus efeitos sobre a qualidade da água. Uma revisão. Boletim do Instituto de Pesca 31(1): 81-88.

Rino, J.A \& Pereira, M.J. 1991. A propósito de Trachelomonas cervicula Stokes (1890) e de Trachelomonas varians Deflandre (1924) (Algae, Euglenophyta), Boletim da Sociedade Broteriana 44: 245-258.

Rosa, Z. M.; Aguiar, C.W. \& Corte-Real, M. 1974. Notas sobre gêneros de algas continentais do Município de Porto Alegre, RS, Brasil. Iheringia Série Botânica 19: 53-62.

Round, F.E. 1983. Biologia das Algas. 2 ed. Rio de Janeiro: Ed. Guanabara Dois, 263p,

Shi, Z.; Wang, Q.; Xie, S. \& Daí, J. 1999. Euglenophyta. Science Press. 414p., il. (Flora algarum sinicarum aquae dulcis, T. 6)

Silva, L.S. 1999. Fitoplâncton de um reservatório eutrófico (lago Monte Alegre), Ribeirão Preto, São Paulo, Brasil. Revista Brasileira de Biologia 59: 281-303.

Starmach, K. 1983. Euglenophyta. In: Starmach, K. (ed.). Flora Slodkowodna Polski. Warszawa: Polska Academia Nauk., v. 3, 593p.

Tell, G. \& Conforti, V.T.D. 1986. Euglenophyta pigmentadas de la Argentina. Stuttgart: J. Cramer. Bibliotheca Phicologica 75: 1-301.

Ter Braak, C. J. F. 1986. Canonical Correspondence Analysis: a new eigenvector technique for multivariate direct gradient analysis. Ecology 67: 1167-1179.

Ter Braak, C. J. F. \& Prentice, I. C. 1988. A theory of gradient analysis. Advances of Ecological Research 18: 271-317.

Tucci, A.; Sant'Anna, C.L.; Gentil, R.C. \& Azevedo, M.T.P. 2006. Fitoplâncton do Lago das Garças, São Paulo, Brasil: um reservatório urbano e eutrófico. Hoehnea 33: 147-175. 